\title{
Linewidth-narrowing mechanism in lasers by nonlinear wave mixing
}

\author{
Moshe Horowitz, Ron Daisy, Baruch Fischer, and John L. Zyskind ${ }^{*}$ \\ Department of Electrical Engineering, Advanced Opto Electronics Research Center, \\ Technion-Israel Institute of Technology, Haifa 32000, Israel
}

Received March 17, 1994

\begin{abstract}
We propose a linewidth-narrowing mechanism in lasers by nonlinear absorptive wave mixing of the counterpropagating beams in the cavity. We give a theoretical analysis and report on a demonstration of the effect with an erbium-doped fiber laser. The system also exhibits bistability in the dependence of the oscillation intensity versus pump power.
\end{abstract}

Coupling between the counterpropagating waves in laser amplifiers gives rise to spatial hole burning and cross saturation effects, which reduce the laser oscillation quality and its coherence. ${ }^{1-3}$ Insight into these effects can be obtained in two ways: first, by consideration of the reflections of the counterpropagating waves from the saturable gain grating that they induce as a result of their interference pattern. These reflections are $180^{\circ}$ out of phase with their corresponding copropagating waves ${ }^{3,4}$ and interfere with them destructively, thus providing a negative feedback. This in turn tends to eliminate the grating by a reduction of the coherence of the waves producing it. Alternatively, it can be verified, as shown below, that in most of the interesting cases a spatially uniform distribution of light intensity in saturable gain media, corresponding to mutually incoherent counterpropagating beams, provides more amplification for the waves that create the saturation than does nonuniform distribution of the gain (i.e., the gain grating induced by the standing-wave pattern of mutually coherent beams with the same intensity average). The reason is that where the intensity is high the saturation decreases the gain, whereas where the gain is unsaturated it is ineffective because the intensity is low. Therefore more gain can be achieved for less coherent waves, as interference is reduced. Moreover, this interference enhances the buildup of a third signal wave with a different frequency (i.e., another mode). Such a signal experiences higher amplification compared with that of the first pair of waves that provides the interference and the nonuniform periodic gain distribution, since the overlap between the intensity and the gain grating patterns is higher. This is a reason for the tendency to multilongitudinal mode oscillation of lasers. In the grating picture this regime corresponds to the situation in which the Bragg condition is not met and the reflections are eliminated.

The main purpose of this Lottor is to present and analyze a mechanism that can be used to flip the sign of the effect of wave mixing, such that it will work in the desired direction of improving the laser oscillation quality and reducing the linewidth. This goal is accomplished by use of a saturable absorber in the laser cavity. Then the nonlinear wave-mixing effect is opposite its role in the saturable gain case. Here the reflections from the induced saturable absorbing grating are in phase (compared with the $180^{\circ}$ phase in the saturable gain case) and provide positive feedback. Similarly, it can be shown that the absorption is lower when the light intensity has a nonuniform periodic distribution along the saturable absorber, compared with that of the uniform case. Therefore interference that produces a standing wave in the cavity will lead to improved coherence and promotion of single longitudinal mode operation. The effect on a third signal wave with a different frequency is also opposite, and the absorption is higher, for the nonuniform distribution case. Here the third wave will be phase mismatched with the induced maxima and minima of the periodic distribution in the saturable and will suffer higher loss. This provides the tendency to eliminate other modes.

Of course, for oscillation to be obtained the cavity must contain an amplifying section. To reduce its deleterious effect on the linewidth, one must suppress the wave mixing in the gain section. This can be done, among other ways, by arranging the cavity such that the polarizations of the counterpropagating beams will be perpendicular in the gain section and parallel in the absorber. The two $\pm 45^{\circ}$ polarization (Faraday) rotators and the polarizer in Fig. 1 satisfy this need.

Saturable absorbers are widely used in laser cavities for passive mode-locking purposes. Their lumped saturation feature drives the laser to produce pulses, where the light energy bunching in the

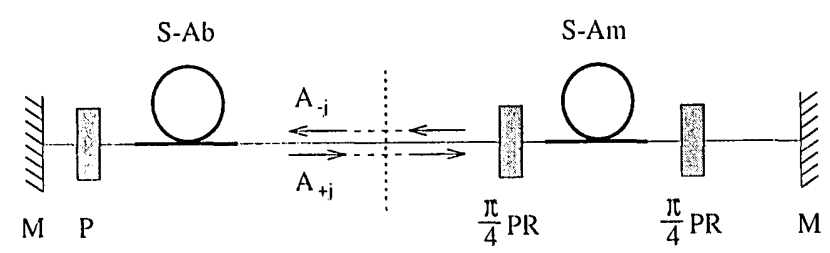

Fig. 1. Laser system with the saturable amplifier (S-Am) and the saturable absorber (S-Ab). PR's, $\pi / 4$ polarization rotators; $\mathrm{P}$, polarizer; M's, mirrors. 
time axis lowers the losses. In the present study we use a distributed saturable absorber and induced wave mixing to enhance periodic bunching in the longitudinal spatial dimension, affecting the frequency domain and driving the laser to narrow linewidths and more coherence. (Previously we showed that a saturable absorber causes spatial bunching and nonuniformity in the transversal plane of an oscillating beam..$^{5}$ Our saturable absorber, the erbiumdoped fiber, cannot be used for passive mode locking because of its long time response $(\sim 10 \mathrm{~ms})$ compared with the cavity round-trip time.

We give here a brief theoretical analysis of the laser system shown in Fig. 1, which in addition to saturable gain includes a saturable absorber. We follow the work by Agrawal and $\mathrm{Lax}^{3}$ and use the intensity-dependent susceptibility $\chi=\chi_{0}(1+$ $i \Omega) /\left(1+\Omega^{2}+I / I_{s}\right)$, which can be taken as a good approximation for the three-level system of erbiumdoped fibers ${ }^{6}$ used in our experiments. $\Omega$ is the frequency detuning from resonance, and $I_{s}$ is the saturation intensity, which for the saturable gain depends on the pumping power. For the gain or the absorption expressions we replace $\chi_{0}$ by positive or negative $\gamma_{0}$, respectively. We extend the analysis of Ref. 3 to multiple $N$ pairs of counterpropagating beams (denoted $j= \pm 1, \pm 2, \ldots \pm N$ ) along the $\pm z$ axis of the cavity, having amplitudes $A_{ \pm j}$, and find the coupled-wave equations

$$
\frac{\mathrm{d} A_{ \pm j}}{\mathrm{~d} z}=\frac{ \pm \gamma_{0}(1+i \Omega)}{2\left(a^{2}-b^{2}\right)^{1 / 2}}\left[A_{ \pm j}-\frac{a-\left(a^{2}-b^{2}\right)^{1 / 2}}{2} \frac{A_{\mp j}}{C_{ \pm j}}\right]
$$

where the plus and the minus signs correspond to the right- and left-propagating beams, respectively, $a=$ $1+\Omega^{2}+\sum_{j}\left(I_{+j}+I_{-j}\right) / I_{s}, I_{ \pm j}=A_{ \pm j} A_{ \pm j}^{*}, C_{+j}=C_{-j}^{*}=$ $\sum_{j^{\prime}} A_{+j^{\prime}}^{*} A_{-j^{\prime}} \exp \left(i \Phi_{j j^{\prime}}\right) / I_{s}, \Phi_{j j^{\prime}}=\Phi_{j j^{\prime}}^{0}+2\left(k_{j}-k_{j^{\prime}}\right) z, k_{j}$ is the wave number, $\Phi_{j j^{\prime}}^{0}$ for $\Omega=0$ is the relative phase at $z=0$ (which depends on the location of the saturable medium in the cavity), and, for $\Omega \neq$ $0, \phi_{j j^{\prime}}=\operatorname{phase}\left(A_{j} A_{-j}^{*}-A_{j^{\prime}} A_{-j^{\prime}}^{*}\right)$ and $b=2\left|C_{ \pm j}\right|$. For the mutually incoherent beams, where the gratings are eliminated, $b=0$.

From Eq. (1) we can obtain the following conclusions: (a) When the counterpropagating beams are mutually incoherent, the second term in the equation vanishes, but the absolute value of the prefactor slightly decreases. In most cases of interest, however, coherence and coupling reduce the absorption or the gain. (b) The influence of one pair of mutually coherent counterpropagating beams (say, $j=1$ ) on another pair $(j=2)$ (which may represent a signal beam and its reflection or another mode in the laser) is given through $b$ and $C_{ \pm j}$ in the equation for $A_{ \pm 2}$ [Eq. (1)]. However, when the two pairs have different frequencies and the Bragg condition is not met, the effect in the second term in the equation with the factor $\exp \left\{i\left[\Phi_{21}^{0}-2\left(k_{2}-k_{1}\right) z\right]\right\}$ is canceled in the integration because of the phase mismatch. Then the prefactor $\gamma_{0} /\left(a^{2}-b^{2}\right)^{1 / 2}$ shows that the existence of a grating induced by the first pair $(b \neq 0)$ raises the absorption or the gain for the signal or a second
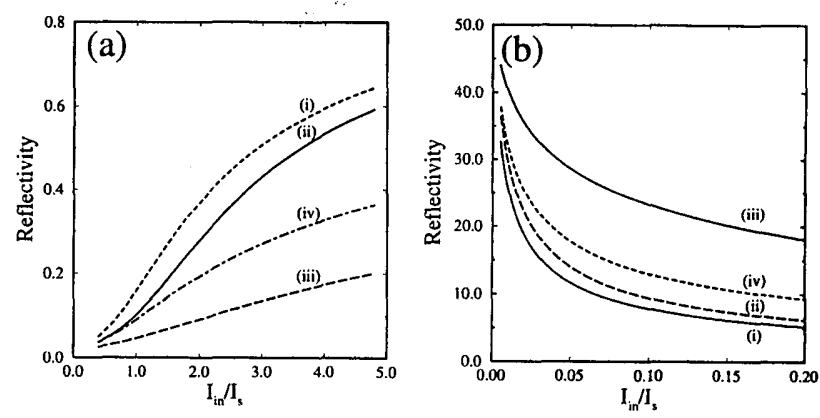

Fig. 2. (a) Reflectivities from the saturable absorber with the mirror (the left half of Fig. 1) with $\gamma_{0} l=-2$ and a mirror reflectivity of $95 \%$ for the following cases: one pair of counterpropagating beams (i) with mutual coupling and interference and (ii) without coupling; (iii) an extra signal beam, affected by the grating of the first pair, where the signal has the same frequency but is $180^{\circ}$ out of phase; and (iv) for a signal out of the Bragg matching frequency. $I_{\text {in }}$ is the intensity of the input beam $I_{-1}$ for (i) and (ii) and is an added small-signal intensity for (iii) and (iv). (b) As in (a) but for the saturable gain case $\left(y_{0} l=2\right)$, and the input here in (i) and (ii) is $I_{+1}$.

mode. (c) For a signal with a degenerate frequency (Bragg matched), the relative phase $\Phi_{21}$ determines whether the signal experiences lower $\left(\Phi_{21}=0\right)$ or higher $\left(\Phi_{21}=180^{\circ}\right)$ absorption or gain. This causes a further elimination (in the absorption case) or promotion (in the gain case) of modes, even in the Bragg regime. One can also obtain a simple understanding from the approximate coupled-wave equation by expanding $\chi(I)$ for $I_{ \pm j} \ll I_{s}$ in a way similar to that in Ref. 4 , in which the role of the grating and the reflections are clearly seen.

For an analysis of the system in Fig. 1, the coupledwave equations are separately solved for the two sections, the amplifier and the absorber, and the effective reflectivity for each part (the right- and left-hand sides of Fig. 1) with its attached mirror is obtained. The amplifier pump power and hence $I_{s}$ are taken to be constant along the gain section. The results in Figs. 2(a) and 2(b) show the main points discussed above: We see the difference between absorbing and gain media; the mutual effect of a pair of beams with and without coupling (for the case in which coupling exists in the gain section, the polarization rotators in Fig. 1 are removed); and the effect of such a pair on another pair (small signal with its reflection, or another mode) without and with Bragg matching for relative phases of $\Phi_{21}^{0}=0^{\circ}$ (which is found to be equivalent to the one-pair reflectivity) and $\Phi_{21}^{0}=180^{\circ}$.

Now we are ready to obtain the oscillation of the full laser system of Fig. 1. We match the two halves of Fig. 1-the input of one section is the output of the other and vice versa-and use the reflectivities for one interacting pair in the absorber part, with or without coupling ${ }^{7}$ and grating, and in the gain part, without coupling and grating. Then we require that the overall gain and losses be equal and obtain the oscillation intensity versus gain. The result, shown in Fig. 3(a), exhibits bistable behavior. As expected, the coupling lowers the threshold and increases the oscillation power. Here we took $\gamma_{0} l=-1.7(l$ 

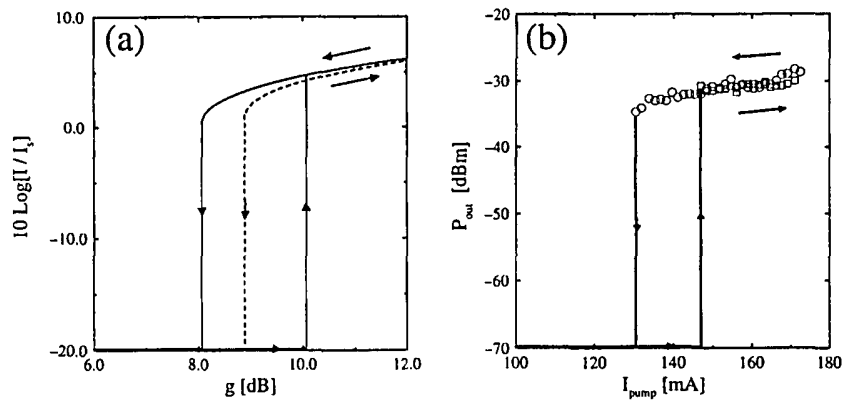

Fig. 3. Theoretical output of the oscillation intensity versus small-signal gain $g$ with (solid curve) and without (dashed curve) coupling of the counterpropagating beams in the saturable absorber. We used $\gamma_{0} l=-1.7$ for the saturable absorber, other losses (due mainly to interfiber couplings) of $67 \%$ for one cavity round trip, and mirror reflectivity of 95\%. (b) Experimental output of the oscillation intensity versus pumping power (with a 980-nm laser). The squares and the circles describe the experimental data for increasing and decreasing $I_{\text {pump }}$, respectively.
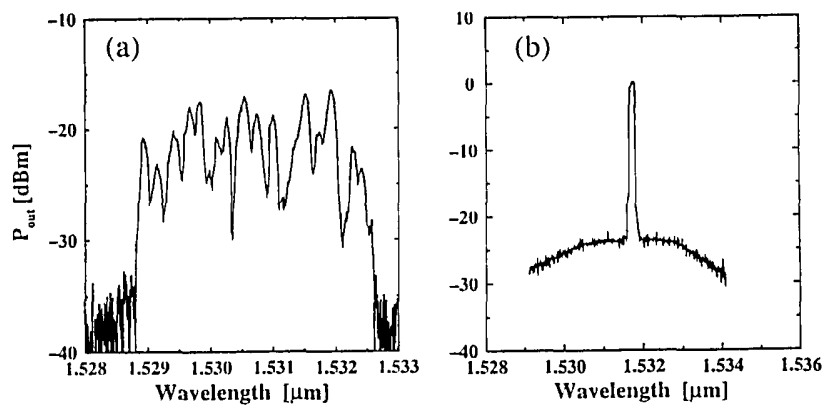

Fig. 4. Spectra of the fiber laser (a) without and (b) with the saturable absorber section.

is the length of the mixing medium) for the saturable absorber sections that fit our experiment, ${ }^{8}$ and $I_{s}$ (amplifier) $=3 I_{s}$ (absorber). We have also taken $\Omega=0$; i.e., the medium is purely absorptive. Similar results however, can be obtained outside this regime, where nonlinear refraction is included.

The experimental demonstration of the saturable absorber line narrowing was carried out with an erbium-doped fiber laser. ${ }^{8}$ Here we give only a brief report on the results and add the experiment with the bistable behavior. The cavity contained two lengths of erbium-doped fiber. One was pumped at $980 \mathrm{~nm}$ to provide gain; the second length was unpumped and functioned as the saturable absorber. Wave mixing in the gain section was eliminated by polarization controllers. The behavior of the oscillation power as a function of the pump power is shown in Fig. 3(b) and, as predicted, exhibits bistable behavior. The switching is sharp with an abrupt transition from the off to the on state and vice versa. The spectra of the fiber laser, with and without the saturable absorber section, are shown in Fig. 4. These measurements are limited by the resolving power of the spectrum analyzer, which was $0.1 \mathrm{~nm}$. The spectrum was stable over time scales of minutes. The specific wavelength, however, is not directed by our filtering mechanism, unless another passive filter is inserted in the cavity. Nevertheless, once the laser starts to operate at a specific line it will tend to stick to it. Other measurements by a rf spectrum analyzer and delayed self-homodyning showed that the laser oscillated mostly in a single longitudinal mode and that the linewidth was ${ }^{8}$ less than $5 \mathrm{kHz}$. We believe, however, that much narrower linewidths can be obtained.

To summarize, we have proposed, analyzed, and demonstrated a linewidth-narrowing mechanism in lasers that uses intracavity nonlinear absorptive wave mixing. The passive nature of the self-induced grating that is distributed along the cavity can provide a special way for obtaining single-wavelength operation, especially of long fiber lasers.

*Permanent address, AT\&T Laboratories, Crawford Hill, 791 Holmdel-Keyport Road, Holmdel, New Jersey 07733.

\section{References}

1. M. Sargent III, M. O. Scully, and W. E. Lamb, Jr., Laser Physics (Addison-Wesley, London, 1977).

2. A. E. Siegman, Lasers (University Science, Mill Valley, Calif., 1986), p. 321.

3. R. P. Agrawal and M. Lax, J. Opt. Soc. Am. 69, 1717 (1979); 71, 515 (1981).

4. B. Fischer, J. L. Zyskind, J. W. Sulhoff, and D. J. DiGiovanni, Opt. Lett. 18, 2108 (1993); Electron. Lett. 29, 1858 (1993).

5. B. Fischer, O. Werner, and M. Horowitz, Appl. Phys. Lett. 58, 2729 (1993).

6. E. Desurvire, J. Lightwave Technol. 8, 1517 (1990).

7. D. G. Steel, R. C. Lind, and J. F. Lam, Phys. Rev. A 23, 2513 (1981).

8. M. Horowitz, R. Daisy, B. Fischer, and J. L. Zyskind, Electron. Lett. 30, 648 (1994). 